\title{
Study on the impulsive pressure of tank oscillating by force towards multiple degrees of freedom
}

\author{
Shigeyuki Hibi ${ }^{1, *}$ \\ ${ }^{1}$ The National Defense Academy, Department of Mechanical Systems Engineering, 1-10-20 Hashirimizu, \\ Yokosuka, Kanagawa 239-8686, Japan
}

\begin{abstract}
Impulsive loads should be excited under nonlinear phenomena with free surface fluctuating severely such as sloshing and slamming. Estimating impulsive loads properly are important to recent numerical simulations. But it is still difficult to rely on the results of simulations perfectly because of the nonlinearity of the phenomena. In order to develop the algorithm of numerical simulations experimental results of nonlinear phenomena are needed. In this study an apparatus which can oscillate a tank by force was introduced in order to investigate impulsive pressure on the wall of the tank. This apparatus can oscillate it simultaneously towards 3 degrees of freedom with each phase differences. The impulsive pressure under the various combinations of oscillation direction was examined and the specific phase differences to appear the largest peak values of pressure were identified. Experimental results were verified through FFT analysis and statistical methods.
\end{abstract}

\section{Introduction}

Impulsive loads[1,2] should be excited under nonlinear phenomena with free surface fluctuating severely such as sloshing and slamming in the field of naval architecture.

Many studies about not only the estimation of the impulsive loads but also elastic behavior of structures have been carried out along with the recent developments of the numerical simulation methods. However the number of experiments to verify those numerical techniques is not enough because of the nonlinearity of the phenomena.

In this study an apparatus which can oscillate a tank by force was introduced in order to investigate impulsive pressure on the wall of the tank. This apparatus can oscillate it simultaneously towards 3 degrees of freedom (up-down, left-right and rotation) with each phase differences. It could happen that larger impulsive pressure is excited under oscillating the tank simultaneously towards multiple degrees of freedom than under single direction oscilation. The author examined the impulsive pressure under the various combinations of oscillation direction and identified the specific phase differences to appear the largest peak values of pressure. Therefor the author showed larger impulsive pressure could appear under the oscillation towards multiple directions in comparison with the oscillation towards a single direction.

In the meantime the uncertainty of the result of experiments has been settled by the GUM[3] (Guide to the Expression of Uncertainty in Measurement) since 1993. Based on this a FFT analysis about measured pressure values was carried out and the confidence interval of them based on $t$-distribution was also determined. At last uncertainty analysis based on the GUM was executed and the reliability of the experiment was assured.

\section{Experimental apparatus measuring instruments}

\subsection{Specification of oscillation apparatus}

The apparatus used in this study can oscillate a tank attached on the bracket harmonically and simultaneously towards 3 degrees of freedom with each phase differences (See Fig. 1 and Table 1).

\footnotetext{
* Corresponding author: hibiteru@nda.ac.jp
} 
The size of tank is $400 \times 400 \times 100(\mathrm{~mm})$ (Height $\times$ Width $\times$ Depth).

We define the symbols for the oscillation directions of 3 degrees of freedom as follows in Fig.2. Heave oscillation (Up - down) stands for $\mathrm{Z}$ and Sway oscillation (Left - right) stands for $\mathrm{X}$ and Roll oscillation (Rotation) stands for $\theta$.
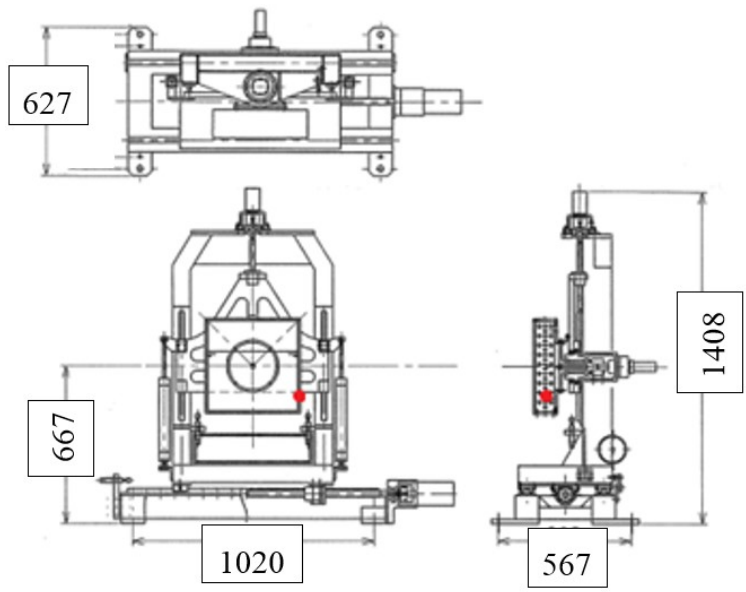

Fig. 1. Oscillation apparatus with 3 degrees of freedom.

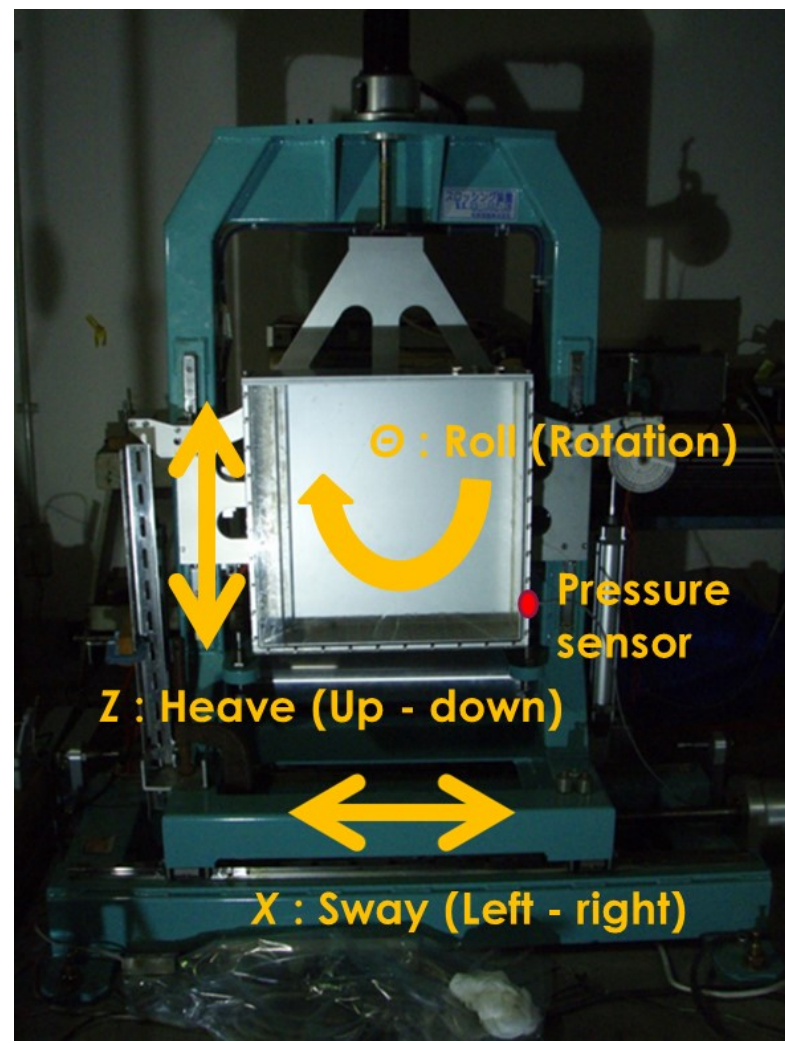

Fig. 2. Oscillation direction and pressure sensor position.
Table 1. Specification of the apparatus about oscillation.

\begin{tabular}{|c|c|c|}
\hline $\begin{array}{c}\text { Direction of } \\
\text { oscillation }\end{array}$ & $\begin{array}{c}\text { Maximum } \\
\text { amplitude }\end{array}$ & $\begin{array}{c}\text { Minimum } \\
\text { period }\end{array}$ \\
\hline $\begin{array}{c}\text { Heave }(\mathrm{Z}) \\
\text { (Up-down) }\end{array}$ & $\pm 50 \mathrm{~mm}$ & $1.0 \mathrm{sec}$. \\
\hline $\begin{array}{c}\text { Sway (X) } \\
\text { (Left-right) }\end{array}$ & $\pm 50 \mathrm{~mm}$ & $1.0 \mathrm{sec}$. \\
\hline $\begin{array}{c}\text { Roll }(\theta) \\
\text { (rotation) }\end{array}$ & $\pm 45 \mathrm{deg}$. & $1.0 \mathrm{sec}$. \\
\hline
\end{tabular}

\subsection{Measuring instruments}

(1) Potentiometer

In order to confirm the acculacy of the roll oscillation control of a servo motor a potentiometer is attached to measure a rotation angle at every moment.

(2) Laser displacement gauge

In order to confirm the acculacy of both heave and sway oscillation control of servo motors laser displacement gauges are placed to measure displacements at every moment.

(3) Pressure sensor

A pressure sensor is attached on the right side of the tank mounted on the apparatus. The position of the sensor is $40 \mathrm{~mm}$ from the bottom. The pressure receiver is a round with a diameter of $8 \mathrm{~mm}$.

\section{Analytical methods for measured data}

\subsection{FFT (Fast Fourier Transform) analysis}

A FFT analysis are applied to the measured pressure data of time history in this study. Frequency components including in each data wave profile are discussed.

\subsection{Determining the confidence intervals based on the $t$-distribution}

Independent randam variables subject to the normal distribution $X_{1}, X_{2}, \ldots X_{N}$ are assumed that mean values are $\mu$ and variances are $\sigma^{2}$ (usually unkonwn). Then sample mean $\bar{X}$ and unbiased variance $u^{2}$ are defined by eq. (1).

$$
\bar{X}=\frac{1}{N} \sum_{i=1}^{N} X_{i}, \quad u^{2}=\frac{1}{N-1} \sum_{i=1}^{N}\left(X_{i}-\bar{X}\right)^{2}
$$


Then randam variable $T$ defined by eq. (2) is subject to Student's $t$-distribution of $N-1$ degree of freedom.

$$
T=(\bar{X}-\mu) /(u / \sqrt{N})
$$

Probability density function of the $t$ - distribution $f_{N-1}(t)$ is given by eq. (3).

$$
f_{N-1}(t)=\frac{\Gamma\left(\frac{N}{2}\right)}{\sqrt{(N-1) \pi} \Gamma\left(\frac{N-1}{2}\right)}\left(1+\frac{t^{2}}{N-1}\right)^{-N / 2}
$$

Here $\Gamma$ is the gamma function.

Therefore in case of a sample of size $N$ the confidence interval at a confidence level $z \%$ is given by eq. (4).

$$
\bar{x}-t_{N-1}(z \%) \frac{u}{\sqrt{N}} \leq \mu \leq \bar{x}+t_{N-1}(z \%) \frac{u}{\sqrt{N}}
$$

\subsection{Uncertainty analysis}

Uncertainty about measuring according to the GUM guidance is evaluated as follows. First of all we determine factors of dispersion in the measuring procedure.

There are 2 types of methods for the estimation of uncertainty in the GUM guidance.

One is called ' $A$ ' type of uncertainty. Its type is based on a statistical method. We estimate uncertainty of each factor using mean values and standard deviations in this type of uncertainty.

The other is called ' $\mathrm{B}$ ' type of uncertainty. Its type is based on another method (e.g. technical information or some experience) without a statistical approach.

After we select the dispersion factors into 2 types of uncertainty, we combine the result to estimate the total uncertainty. the expanded uncertainty $U$ is obtained by multiplying the combined standard uncertainty $u_{\mathrm{c}}(\mathrm{y})$ by a coverage factor $k(k=2$. This means the $95 \%$ confidence level ).

\section{Experiment and discussion}

\subsection{Investigating phase differences during simultaneous oscillation towards 2 degrees of freedom}

The reliability of oscillation amplitude towards each degree of freedom is assured even with a period close to the natural period of the tank by previous study.

In this study we chose 3 pairs of 2 degrees of freedom from 3 one. Those will be subscribed as follows $(\theta-X, \theta-Z, X-Z)$. The oscillation period is uniformly 1.08 (sec.) which is close to the natural period of the tank in case of the water depth is $60 \mathrm{~mm}$ (fixed). The oscillation amplitudes are $15 \mathrm{~mm}$ for $X$ and $Z$ and 20 degrees for $\theta$

We measured time historys of pressure on the wall of the tank repeatedly while changing the phase difference from 0 degree to 360 degrees. We identified the phase difference which cause the largest peak value of pressure for each oscillation pair $(\theta-X, \theta-Z, X-Z)$. The results are shown in Table 2 .

Table 2. Phase difference which cause the largest peak value.

\begin{tabular}{|c|c|}
\hline Oscillation pair & Phase difference \\
\hline$\theta-X$ & 15 degrees \\
\hline$\theta-Z$ & 335 degrees \\
\hline$X-Z$ & 220 degrees \\
\hline
\end{tabular}

Example results of time history of pressure for each oscillation pair are shown in Figs. 3(a), (b) and (c). In any cases it can be deduced that there is periodicity in the pressure fluctuation, but it is found that the peak values have variations.

\subsection{FFT analysis for measured time history of pressure}

FFT analysis was carried out with 1,12 and 19 period intervals from the measured data of time history of pressure for each oscillation pair. The results are shown in Figs. 4(a), (b) and (c). The waveform of the spectrum for 1 period 
interval contains the ones for 12 and 19 period intervals. We can also deduce that although the obtained

peak values varies, the waveform itself seems periodic.



Fig. 3(a). Time history of pressure about $\theta-X$ oscillation.

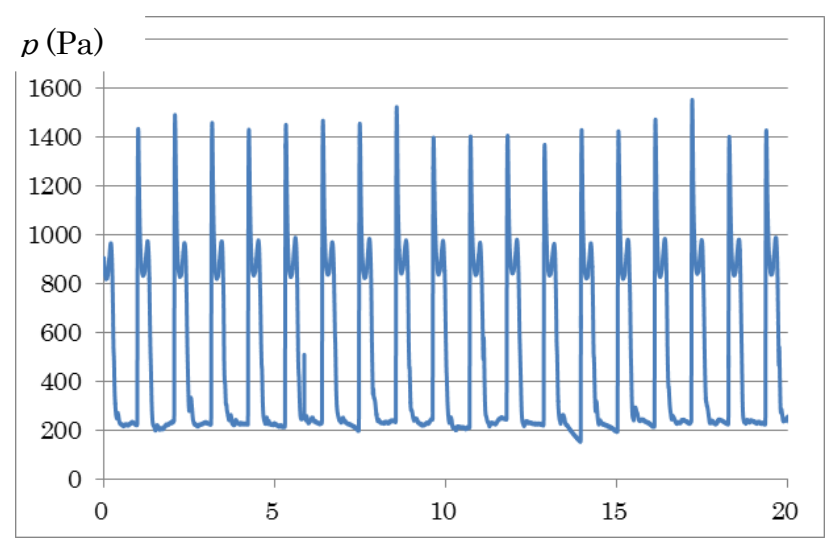

Fig. 3(b). Time history of pressure about $\theta-Z$ oscillation.

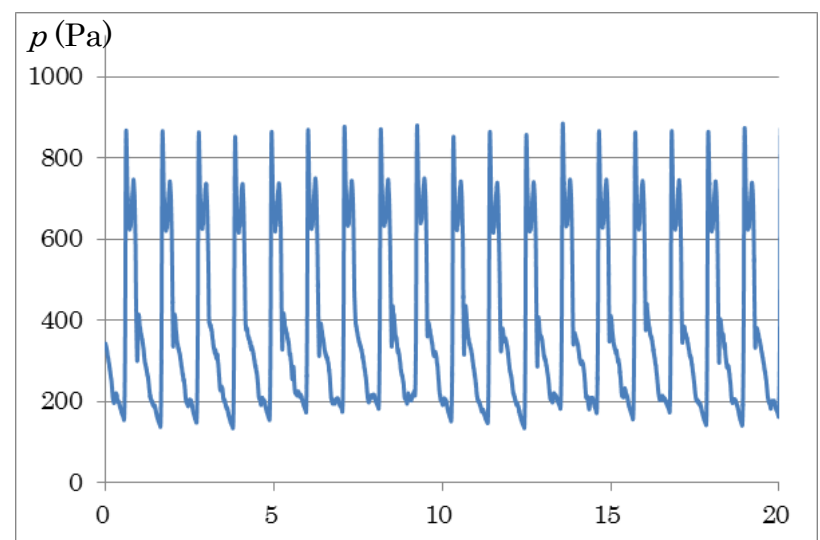

Fig. 3(c). Time history of pressure about $X-Z$ oscillation.

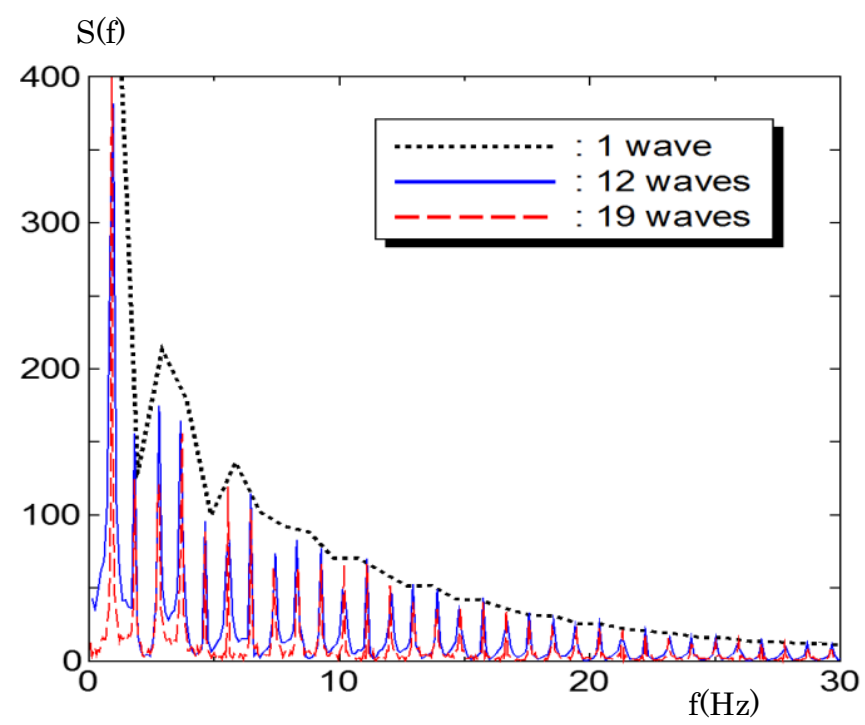

Fig. 4(a). FFT analysis about $\theta-X$ oscillation.

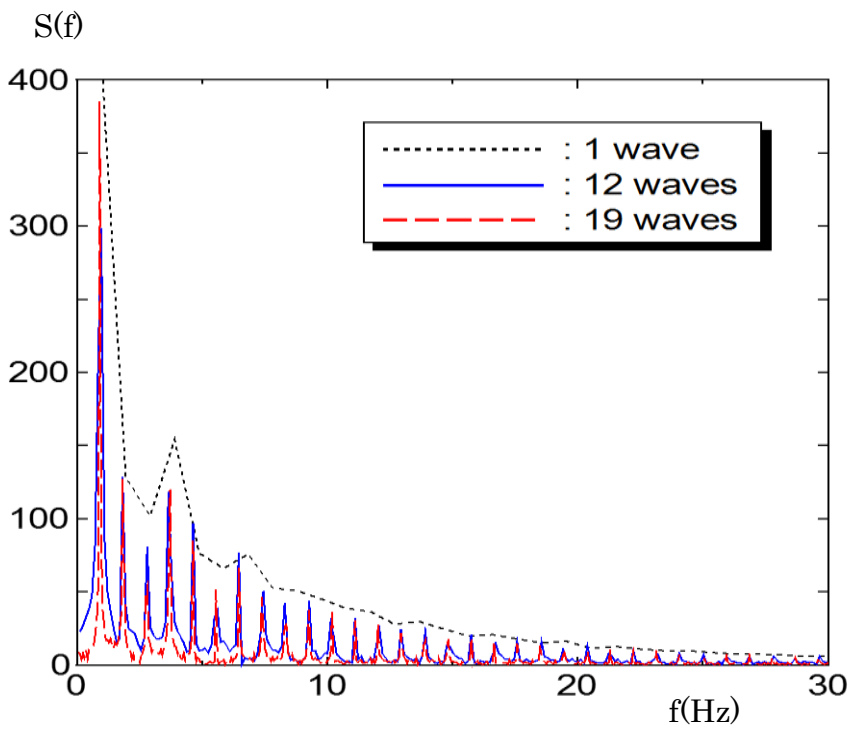

Fig. 4(b). FFT analysis about $\theta-Z$ oscillation.

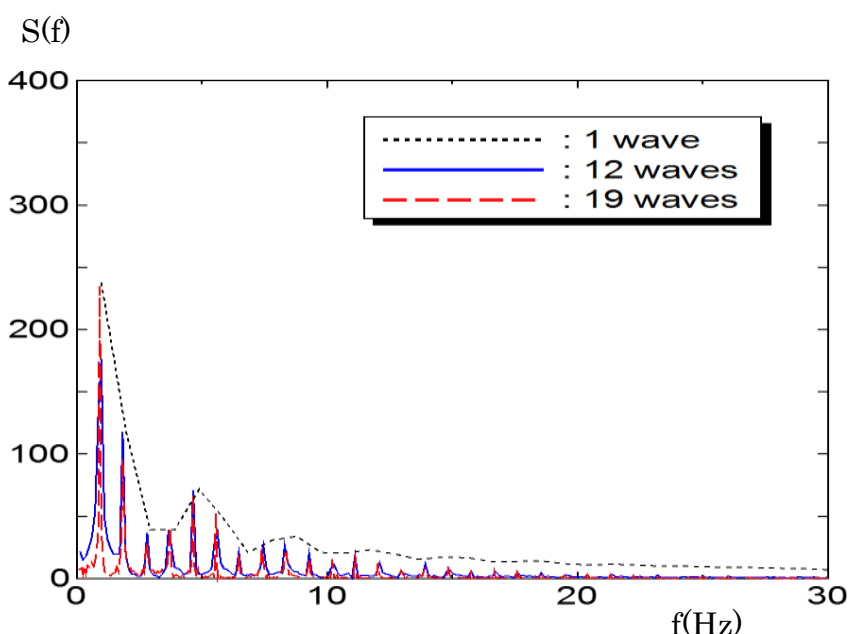

Fig. 4(c). FFT analysis about $X-Z$ oscillation. 


\subsection{Estimation of confidence intervals for the confidence level $95 \%$}

Experiments were repeated as one measurement for 20 seconds. Peak values were extracted from the time history of pressure for each oscillation pair. Next confidence intervals for the confidence level $95 \%$ were determined. The results are shown in the Fig. 4.

Although the width of the confidence interval tends to decrease when the number of samples increases, it is considered that there is a significant width, which may indicate the nonlinearity of the present experimental data.

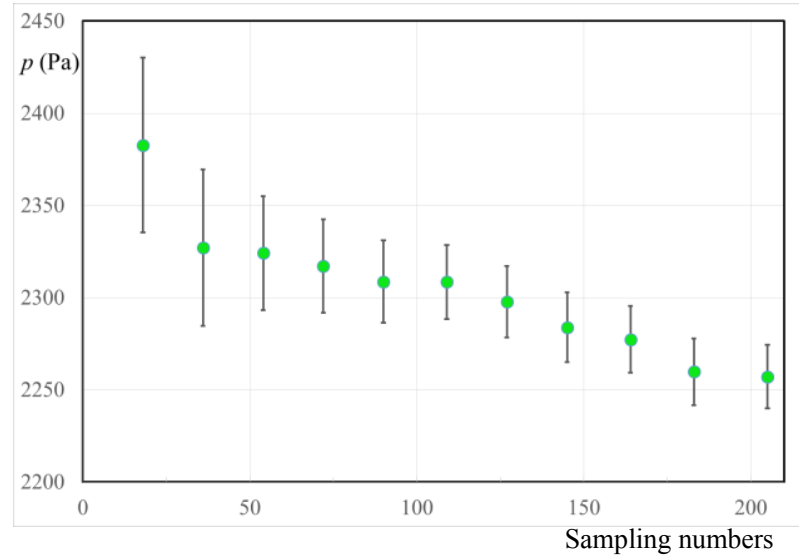

Fig. 5(a). Estimation of peak values at confidence level 95\% about $\theta-X$ oscillation.

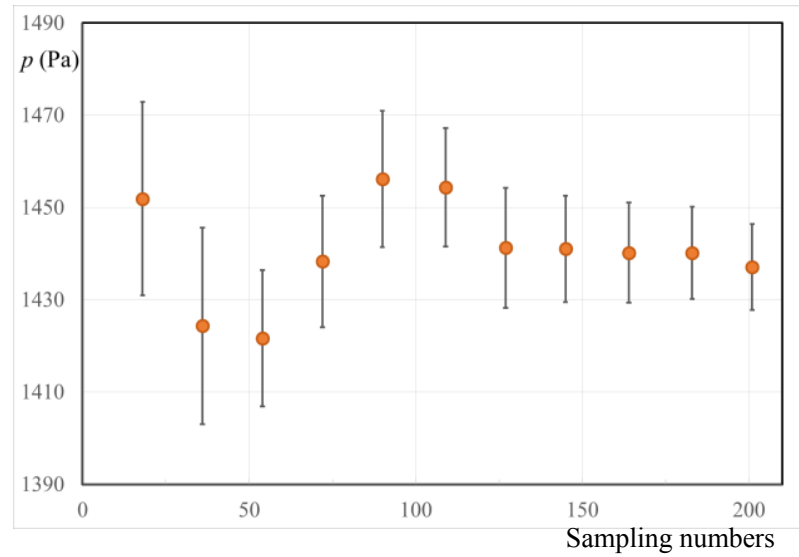

Fig. 5(b). Estimation of peak values at confidence level 95\% about $\theta-Z$ oscillation.

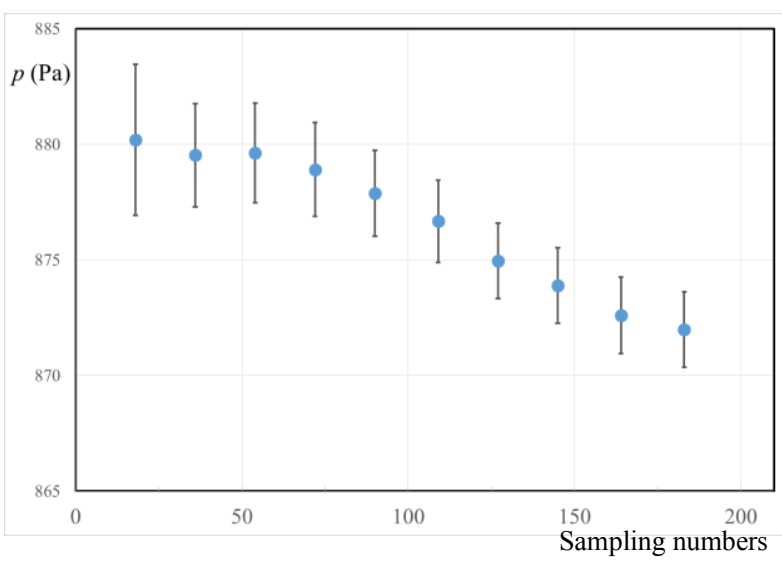

Fig. 5(c). Estimation of peak values at confidence level 95\% about $\mathrm{X}-Z$ oscillation.

\subsection{Determining the expanded uncertainty}

Expanded uncertainty was determined according to the budget table shown in Table 3 . The function $f$ used to estimate the uncertainty when measuring pressure values is in eq. (5).

$$
f=\rho g a(V(d)-V(20))
$$

Where $\rho$ is the density of water and $\mathrm{g}$ is the gravitational acceleration. ' $a$ ' is the coefficient for conversion from voltage to pressure. $V(\mathrm{~d})$ is the voltage value of an amplifier at the $d \mathrm{~mm}$ water depth and $V(20)$ is the voltage value of an amplifier at the $20 \mathrm{~mm}$ water depth.

The expanded uncertainty calculated from Table 3 is $101.9(\mathrm{~Pa})$. The number of iterations used here is 11 times. In most cases it can be seen that the peak value varies around a mean value at this interval.

\section{Conclusion}

In this study impulsive pressure on the tank wall were measured and some information were found about it as below.

- The fluctuation measured had some periodism but the peak values had variation.

- From the estimation of confidence intervals for the measured values at the confidence level $95 \%$ significant intervals were found to exist.

- Since characteristics of impulsive pressure for the oscillation directions of 2 degrees of freedom is known, confirmation of that for the oscillation directions of 3 degrees of freedom is a future task. 


\section{References}

1. Hakan Akyildiza, Erdem U“nal, Ocean Engineering 32, 1503 (2005)

2. P. Temarel, W. Bai, A. Bruns, Q. Derbanne, D. Dessi,S. Dhavalikar, N. Fonseca, T. Fukasawa, X. Gu, A. Nestegård,A. Papanikolaou, J. Parunov, K.H. Song m, S. Wang, Ocean Engineering 119, 274 (2016)
3. JCGM2008, Evaluation of measurement data Guide to the expression of uncertainty in measurement, (2008)

Table 3. Budget table for the estimation of uncertainty

\begin{tabular}{|c|c|c|c|c|c|c|c|c|c|}
\hline \multicolumn{2}{|c|}{ Factors of uncertainty : $x_{i}$} & $\begin{array}{c}\text { Cause of } \\
\text { uncertainty }\end{array}$ & $\begin{array}{c}\text { Type of } \\
\text { uncertainty }\end{array}$ & $\begin{array}{c}\text { Shapes of } \\
\text { probabilistic distribution }\end{array}$ & \begin{tabular}{|l|} 
Mean \\
value
\end{tabular} & $\begin{array}{ll}\begin{array}{l}\text { Sensitivity } \\
\text { factor }\end{array} & \frac{\partial f}{\partial x_{i}}\end{array}$ & $\begin{array}{c}\text { Standard } \\
\text { uncertainty }\end{array}$ & & \\
\hline$a$ & $\begin{array}{l}\text { Coefficient for conversion } \\
\text { from voltage to pressure }\end{array}$ & Repeated experiment & A & Normal & 159.0 & 3.888 & 0.8828 & & \\
\hline$V(20)$ & $\begin{array}{l}\text { Voltage value at the } \\
20 \mathrm{~mm} \text { water depth }\end{array}$ & Repeated experiment & A & Normal & 2.815 & 1558 & 0.03199 & & \\
\hline$V p$ & $\begin{array}{l}\text { Calibration of } \\
\text { the pressure sensor }\end{array}$ & $\begin{array}{l}\text { Calibration error of } \\
\text { the pressure sensor }\end{array}$ & B & Uniform & 2.418 & 1 & 4.35 & & \\
\hline Vamp & $\begin{array}{l}\text { Calibration of } \\
\text { the amplifier }\end{array}$ & $\begin{array}{c}\text { Calibration error } \\
\text { of the amplifier }\end{array}$ & B & Uniform & 5.00 & 1 & 9.00 & & \\
\hline$u_{c}$ & $\begin{array}{c}\text { Combined } \\
\text { standart uncertainty }\end{array}$ & & & & & & & 50.96 & (Pa) \\
\hline$u$ & $\begin{array}{l}\text { Expanded } \\
\text { uncertainty }\end{array}$ & & & & & & & 101.9 & (Pa) \\
\hline
\end{tabular}

